\title{
Nuevo subtipo de acondrogénesis familiar tipo IA (Houston-Harris)
}

\author{
New subtype of familial achondrogenesis type IA (Houston-Harris)
}

\begin{abstract}
Sergio Alberto Ramírez-García1, Diana García-Cruz²*, Iván Cervantes-Aragón², Wadih Emilio Bitar-Alatorre ${ }^{3}$, Ingrid Patricia Dávalos-Rodríguez ${ }^{4}$, Nory Omayra Dávalos-Rodríguez², Jorge Román Corona-Rivera ${ }^{5}$

y José Sánchez-Corona ${ }^{6}$

${ }^{1}$ Universidad de la Sierra Sur, Instituto de Investigaciones sobre la Salud Pública, Sistema de Universidades Estatales de Oaxaca, Miahuatlán de Porfirio Díaz, Oax.; ' Instituto de Genética Humana Dr. Enrique Corona Rivera, Departamento de Biología Molecular y Genómica, Centro Universitario de Ciencias de la Salud, Benemérita Universidad de Guadalajara; ${ }^{3}$ Traumatología y Ortopedia, Hospital Ángeles del Carmen; ${ }^{4}$ División de Genética, Centro de Investigación Biomédica de Occidente, Instituto Mexicano del Seguro Social; ${ }^{5}$ Servicio de Pediatría, Nuevo Hospital Civil Dr. Juan I. Menchaca; ${ }^{6}$ Medicina Molecular, Centro de Investigación Biomédica de Occidente, Instituto Mexicano del Seguro Social. Guadalajara, Jal. México
\end{abstract}

\section{Resumen}

Introducción: La acondrogénesis es una displasia esquelética que se caracteriza principalmente por talla baja, micromelia grave, tórax corto y estrecho, prematurez, polihidramnios, hidropesía fetal y muerte fetal in utero o neonatal. Según los hallazgos radiológicos e histopatológicos existen tres tipos de acondrogénesis: tipo 1A (Houston-Harris), tipo 1B (Fraccaro) y tipo 2 (Langer-Saldino). Caso clínico: Se sometió a estudio a un producto femenino prematuro cuyas características clínicas, radiológicas e histopatológicas fueron compatibles con acondrogénesis tipo 1A. La información familiar permitió concluir que los cuatro productos de los seis embarazos previos se encontraban afectados. El análisis estadístico en por lo menos cuatro familias previamente descritas, incluyendo este caso familiar, mostró diferencias significativas entre el número de miembros esperado y el observado, siendo incongruente con el modo de herencia autosómico recesivo previamente reportado. Conclusiones: Podría considerarse un nuevo subtipo de acondrogénesis tipo $1 \mathrm{~A}$ debida a la presencia de una mutación germinal preferencial.

PALABRAS CLAVE: Acondrogénesis tipo 1A. Mutación germinal. Displasia esquelética.

\begin{abstract}
Background: Achondrogenesis is a skeletal dysplasia characterized primarily by short stature, severe micromelia, short and narrow chest, prematurity, polyhydramnios, fetal hydrops, and in utero or neonatal death. Based on the radiological and histopathological findings, there are three types of achondrogenesis: type $1 A$ (Houston-Harris), type $1 B$ (Fraccaro) and type 2 (Langer-Saldino). Clinical case: $A$ premature female product was studied whose clinical, radiological and histopathological characteristics were compatible with achondrogenesis Type $1 A$. The family information allowed us to conclude that the 4 products of the 6 previous pregnancies were affected. Statistical analysis in at least 4 families previously described, including this family case showed significant differences between expected and observed number of members, being incongruent with an autosomal recessive mode of inheritance previously reported. Conclusions: therefore, it could be considered a new subtype of achondrogenesis type $1 A$ due to the presence of a preferential germline mutation.
\end{abstract}

KEY WORDS: Achondrogenesis type 1A. Germinal mutation. Skeletal dysplasia.

\author{
Correspondencia: \\ *Diana García-Cruz \\ Sierra Mojada 950, edificio P, Segundo nivel \\ Col. Independencia \\ C.P. 44340 Guadalajara, Jal., México \\ E-mail: Dianagarcr@ hotmail.com
}

Fecha de recepción: 19-12-2017

Fecha de aceptación: 14-02-2018

DOI://dx.doi.org/10.24875/CIRU.M18000008
Cir Cir. 2018;86:89-98

Disponible en PubMed www.cirugiaycirujanos.com 


\section{Introducción}

Las displasias esqueléticas son un grupo heterogéneo de trastornos con un desarrollo anormal del esqueleto (en el tamaño y la forma de las extremidades, el tronco y el cráneo). Se ha designado como osteocondrodisplasia a las que resultan de problemas en el proceso normal de osificación $n^{1,2}$. En este grupo encontramos las acondrogénesis (ACG), las cuales son identificables durante la vida fetal 0 al nacimiento, y se acompañan de polihidramnios, hidropesía fetal e hipoplasia pulmonar que ocasionan mortalidad neonatal o mortinatos $^{3}$.Los pacientes con estas displasias presentan cuello y tronco cortos, tórax estrecho, abdomen prominente, talla baja, micromelia grave y dismorfia facial (exoftalmia, puente nasal plano, nariz corta con narinas antevertidas, orejas de implantación baja y protrusión lingual). Los hallazgos radiológicos principales son ausencia de osificación o gravemente reducida de los huesos de la bóveda craneana y de los cuerpos vertebrales del sacro, y costillas cortas con orientación horizontal. El ilion se encuentra acortado, en forma de media luna. Los huesos largos son pequeños, arqueados y ocasionalmente estrellados, con espolones metafisarios orientados longitudinalmente ${ }^{3,4}$.

A la fecha se han descrito tres tipos de ACG: tipo IA (ACG1A, Houston-Harris; MIM \#200600), causado por la mutación del gen TRIP11 locus 14q32; tipo IB (ACG1B, Fraccaro; MIM \#600972), por la mutación del gen SLC26A2 locus $5 q 32$ (ambos tipos se describen con modo de herencia autosómico recesivo; y tipo II (ACG2, Langer-Saldino; MIM \#200610), debido a la mutación del gen COL2A1 locus 12q13.11, con un patrón autosómico dominante. La ACG tiene una frecuencia global de 1-50,000. Para el caso de la ACG1A, se han reportado mutaciones en el gen TRIP11, pero no son concluyentes como causa única ${ }^{3-8}$. Estas tres variantes son letales in utero. Las características radiológicas e histopatológicas permiten el diagnóstico diferencial; entre los tipos I y II es más fácil, ya que son clínicamente diferentes ${ }^{3-6}$. El presente trabajo se propone ser el primer reporte de un nuevo subtipo de ACG1A, con un modo de herencia previamente no reportado, que no es autosómico recesivo, y hallazgos histopatológicos no observados previamente.

\section{Caso clínico}

Recién nacida producto de la séptima gestación. Padres no consanguíneos (Fig. 1). Desde la semana
12 de gestación los ultrasonogramas abdominales revelaron extremidades cortas. Desde la semana 28 presentaba hipoactividad fetal, con probable hidropesía y polihidramnios grave. El producto fue obtenido a las 30 semanas mediante cesárea. Al nacimiento presentó un peso de $1600 \mathrm{~g}$, talla talón-sutura coronal de $23 \mathrm{~cm}$, perímetro cefálico de $22 \mathrm{~cm}$, perímetro torácico de $18.5 \mathrm{~cm}$, perímetro abdominal de $21 \mathrm{~cm}$ y un segmento inferior de $6 \mathrm{~cm}$, enanismo rizomélico y mesomélico, macrocránea, leve hipertelorismo ocular, implantación baja de los pabellones auriculares, puente nasal corto, nariz corta y ancha, microrretrognatia, cuello corto, hipertricosis dorsal toraco-lumbar, importante acortamiento del tronco con hipoplasia costal, abdomen prominente y micromelia de las cuatro extremidades. La paciente fallece a los 15 minutos después del nacimiento.

\section{Estudio familiar y genético}

Los padres sanos, de 35 años (padre) y 33 años (madre) años al nacimiento del caso. Se realizó cariotipo con bandeo GTG de los padres, resultando normal en ambos. El análisis genealógico reveló la presencia de otros hermanos afectados, con las mismas características clínicas II-1, II-2, II-3, II-5 y II-7; II-4 fue un aborto; II-6 fue clínica y radiológicamente sana (fig. 1). Las características clínicas de los familiares se muestran en la tabla 1.

\section{Estudios imagenólogicos}

Los hallazgos radiológicos de la serie ósea completa se muestran en la tabla 2 y la figura $2 \mathrm{~B}$. Desde el aspecto general se observa pobre osificación de los huesos del esqueleto axial y apendicular, con predominio en los cuerpos vertebrales, hipoplasia de los huesos de la pelvis y acortamiento de los huesos largos de las cuatro extremidades, con encorvamiento de estas.

\section{Estudio anatomopatológico}

La descripción macroscópica reportó maceración extensa, ascitis y edema generalizados (Fig. 2). El estudio histopatológico de la epífisis del fémur por biopsia se realizó utilizando ácido fórmico y embebido en parafina; en el aspecto macroscópico reveló un hueso descalcificado. Se realizaron diferentes cortes histológicos con un grosor de $7 \mathrm{~mm}$, que fueron 


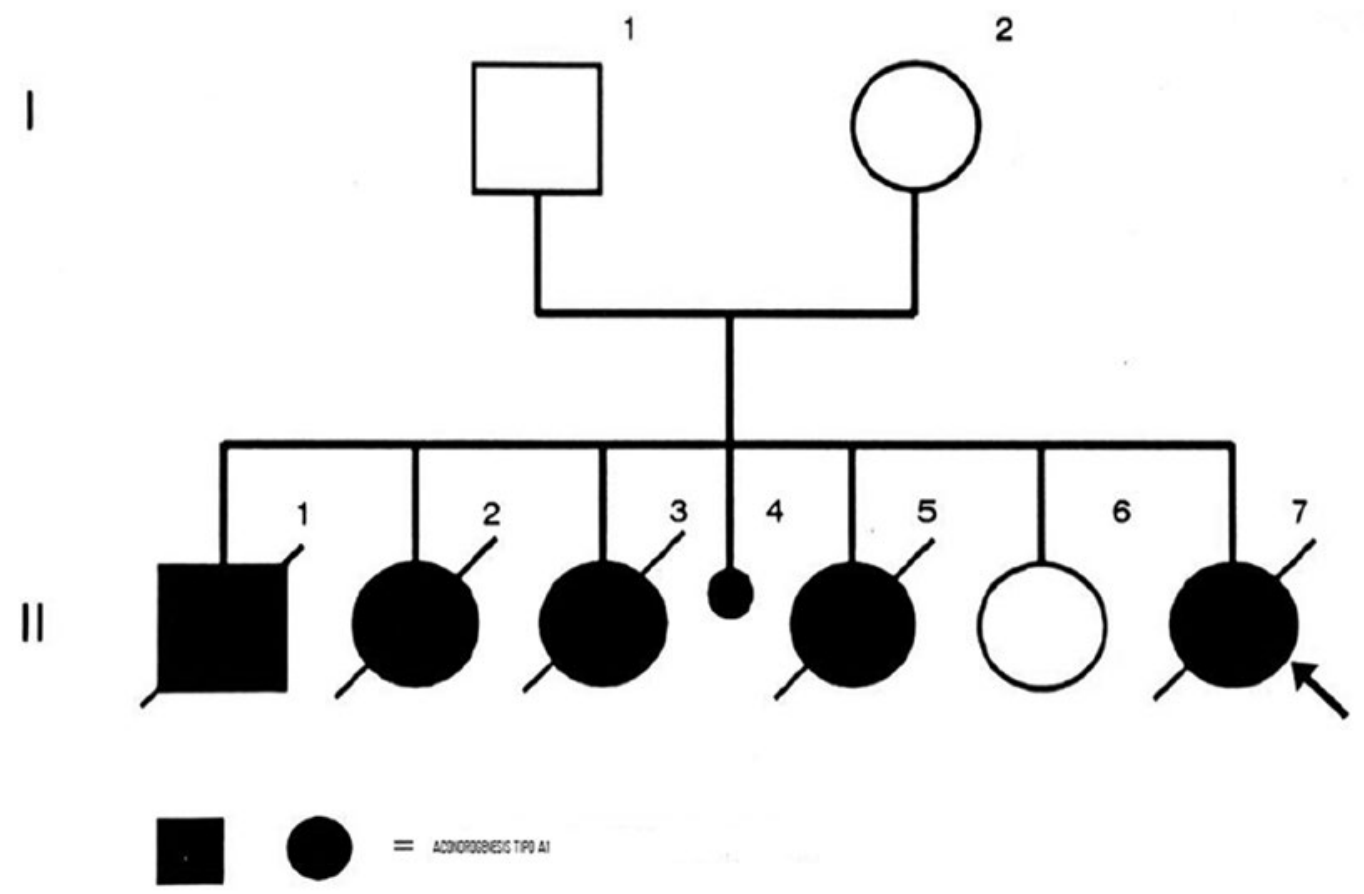

Figura 1. Árbol genealógico.
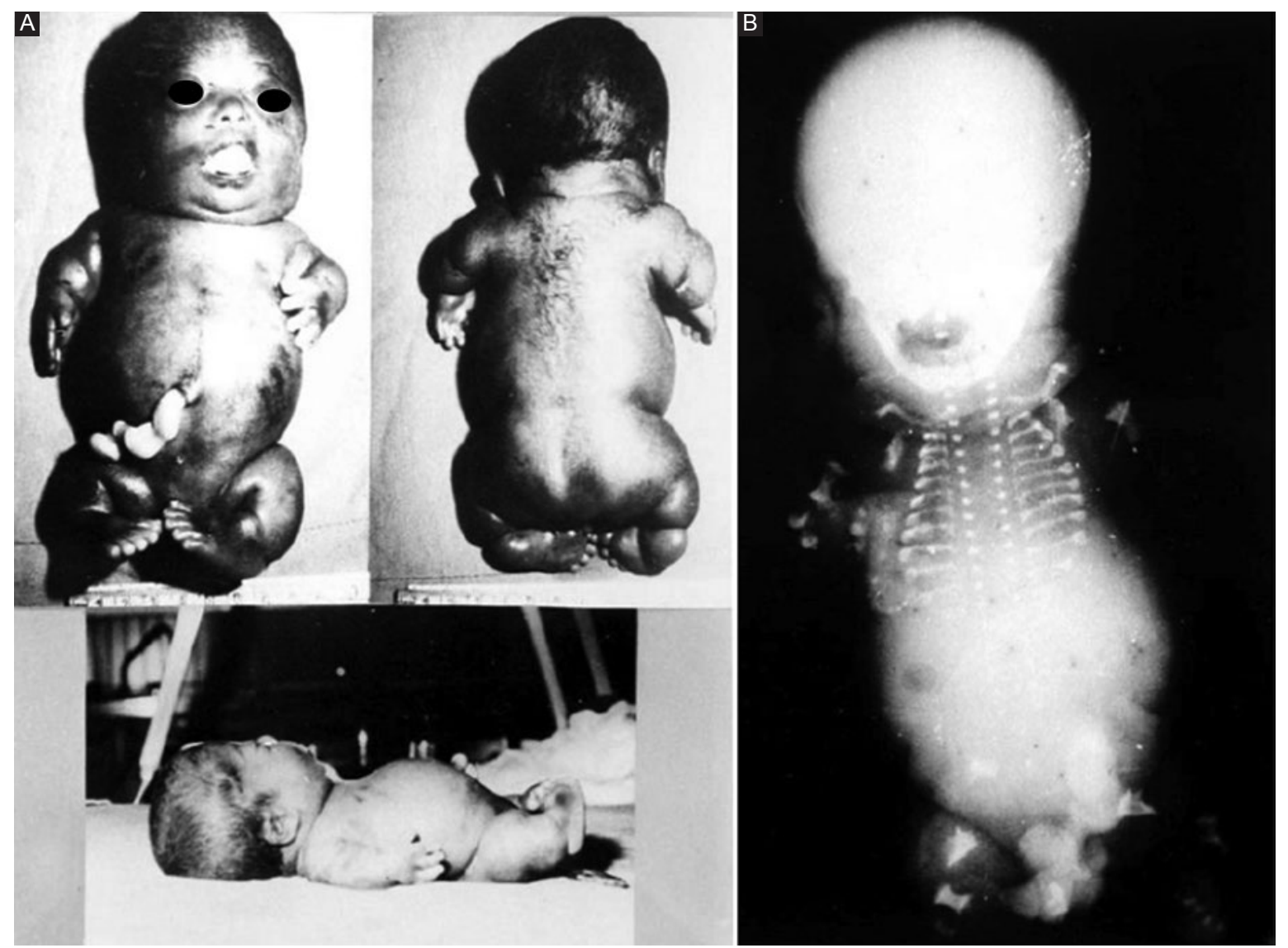

Figura 2. A: foto de la paciente post mortem. Obsérvense la apariencia hidrópica y el cuello, el tórax y las extremidades cortas. B: fotografía de los rayos $X$ de la paciente. Nótense la pobre osificación del cráneo y las vértebras, las costillas cortas, la pelvis hipoplásica, el ilion arqueado y el fémur en forma de cuña. 


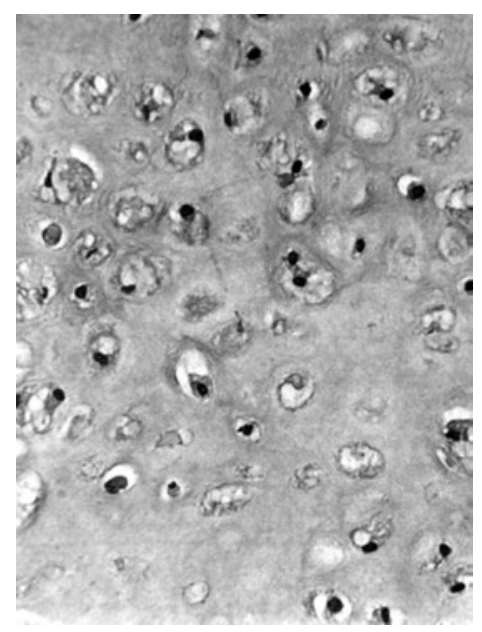

Figura 3. Biopsia del fémur. Obsérvense los condrocitos vacuolados.

teñidos con hematoxilina/eosina, azul alciano/PAS y tricrómica de Masson, y analizados mediante microscopía de luz. Los resultados fueron: matriz cartilaginosa normal, con condrocitos escasos, densamente empaquetados y vacuolados, PAS positivos, y algunos condrocitos que dan un aspecto de ojos de toro (al centro de la biopsia con azul alciano/PAS; Fig. 3); lagunas óseas alargadas y una zona pseudomesenquimatosa entre el cartílago y el hueso. No se encontraron cuerpos de inclusión intracondrocíticos y se halló cartílago extraesquelético involucrado.

\section{Discusión}

En la proposita, así como en varios integrantes de la familia, se encontraron los hallazgos patognomónicos de ACG1A, como polihidramnios en el segundo trimestre de gestación y las anormalidades radiológi-

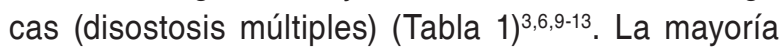
de los casos son prematuros y nacidos muertos; algunos permanecen vivos por minutos después del nacimiento, como en el presente caso ${ }^{7}$. La información de la genealogía analizada permitió concluir que los cuatro productos de los seis embarazos previos se encontraban afectados clínicamente con ACG1A. La proposita estaba más afectada (presentaba una dismorfia facial grave) en comparación con su hermano que falleció con el mismo problema; esto se debe al hydrops fetalis y el polihidramnios, que son causantes de malformaciones.

El diagnóstico diferencial de ACG1A se hace descartando condiciones de dos grupos nosológicos de enfermedades que presentan alta mortalidad perinatal: los defectos cromosómicos que producen dismorfias faciales y malformaciones, las cuales fueron descartadas, ya que el estudio citogenético en ambos padres, fue normal; y las displasias esqueléticas letales, entre ellas la displasia diastrófica MIM \#222600 (causada por mutaciones en el gen SLC26A2 locus $5 q 32)$, síndromes torácicos de costillas cortas con 0 sin polidactilia (existen varios tipos con patrones de herencia autosómica dominante o recesiva), la osteogénesis imperfecta tipo II, MIM \#166210, con patrón autosómico dominante, causada por mutaciones en el gen COL1A2 locus $7 q 21,3$ y por el gen COL1A1 locus 17q21.33), la displasia pélvica de configuración «Sonaja de bebé», MIM \#605838 (todavía no tiene un gen candidato), la fibrocondrogénesis tipo 1, MIM \#228520 (con un patrón autosómico recesivo causado por mutaciones en COL11A1 locus 1p21.1), y tipo 2, MIM \#614524 (con herencia tanto autosómica dominante como recesiva, causada por mutaciones en COL11A2 locus 6p21.32), la displasia tanatofórica MIM \#187600 (causada por mutaciones en el gen FGFR3 locus 4p16.3), y especialmente la ACG2, por el patrón de trasmisión autosómico dominante, similar al que se presenta en la familia analizada ${ }^{8-13}$.

El diagnóstico se puede realizar también por secuenciación. Para los pacientes con ACG2, las mutaciones se encuentran en COL2A16,10; en la ACG1B, en el gen DTDST ${ }^{3,5,9,12-17}$. En la búsqueda de un gen implicado para ACG1A, Smits, et al..$^{13}$ encontraron que los ratones con la deficiencia de GMAP-210 morían con un fenotipo similar a ACG1A. Este gen es homólogo al TRP11 en el humano, en el que se han encontrado 11 mutaciones (la mayoría en estado heterocigoto compuesto) ${ }^{13}$. Al parecer, dichas mutaciones se heredan con un patrón de herencia autosómico recesivo, y como los afectados son heterocigotos compuestos, se especula que llegan a permanecer vivos por minutos o días después del nacimiento, ya que los homocigotos son incompatibles con la vida, por lo cual son poco frecuentes, como sucede en la displasia tanatofórica ${ }^{14-16}$. Grigelioniene, et al. ${ }^{4}$ también muestran evidencia sobre el patrón autosómico recesivo, y en contraparte encuentran gemelos dicigóticos con un fenotipo clínico y radiológico de ACG1A con genotipo homocigoto para una nueva mutación sin sentido en TRIP114. También encontraron un paciente heterocigoto compuesto para una mutación sin sentido y una deleción del extremo 3' del gen, con manifestaciones más leves. Por tanto, las mutaciones del gen TRIP11 pueden abarcar un rango fenotípico más amplio, pero hasta la fecha no son causa única de $A C G 1 A^{13}$, como vemos en la familia de la proposita analizada en este reporte, ya que clínicamente 
Tabla 1. Datos clínicos de los hermanos de la proposita

\begin{tabular}{|c|c|c|c|c|c|c|}
\hline Embarazo & $\|-1$ & II-2 & II-3 & II-4 & $\| l-5$ & II-6 \\
\hline Sexo & Masculino & Femenino & Femenino & $\begin{array}{l}\text { No } \\
\text { determinado }\end{array}$ & Femenino & Femenino \\
\hline $\begin{array}{l}\text { Edad gestational } \\
\text { en semanas }\end{array}$ & 29 & $23 / 24$ & 28 & 8 & 30 & 39 \\
\hline $\begin{array}{l}\text { Hallazgos } \\
\text { clínicos }\end{array}$ & $\begin{array}{l}\text { Extremidades } \\
\text { cortas }\end{array}$ & $\begin{array}{l}\text { Múltiples } \\
\text { malformaciones }\end{array}$ & $\begin{array}{l}\text { Labio leporino, } \\
\text { nariz corta y plana, } \\
\text { cuello corto, tórax } \\
\text { y extremidades, } \\
\text { hidropesía fetal }\end{array}$ & $\begin{array}{l}\text { No aplica, } \\
\text { fue aborto }\end{array}$ & $\begin{array}{l}\text { Labio leporino, nariz corta y } \\
\text { plana, cuello corto, tórax y } \\
\text { extremidades, hidropesía fetal }\end{array}$ & Normal \\
\hline $\begin{array}{l}\text { Edad de } \\
\text { muerte/horas } \\
\text { continuadas }\end{array}$ & 4 & 2 & 2 & No aplica & 2 & No aplica \\
\hline $\begin{array}{l}\text { Talla al } \\
\text { nacimiento }(\mathrm{cm})\end{array}$ & No recordado & No recordado & No recordado & No aplica & 23 & 50 \\
\hline $\begin{array}{l}\text { Peso al } \\
\text { nacimiento (g) }\end{array}$ & No recordado & 400 & No recordado & No aplica & 650 & 3000 \\
\hline Polihidramnios & $\begin{array}{l}\text { Dato } \\
\text { desconocido }\end{array}$ & $\begin{array}{l}\text { Dato } \\
\text { desconocido }\end{array}$ & Presente & Ausente & Presente & Ausente \\
\hline Placenta & $\begin{array}{l}\text { Dato } \\
\text { desconocido }\end{array}$ & Degeneración & Dato desconocido & $\begin{array}{l}\text { Dato } \\
\text { desconocido }\end{array}$ & Edamatizada e hipocromica & Normal \\
\hline
\end{tabular}

corresponden a casos con ACG1A, pero la genealogía no es congruente con un patrón de herencia autosómico recesivo.

Derivado de lo anterior, se analizaron la frecuencia de miembros esperados $(E)$ y observados $(O)$ afectados en tres familias con características clínicas de ACG1A reportadas por Borochowitz, et al. ${ }^{17}$ (Tabla 1), junto con la del presente reporte $(n=22 ; E=6$ y $O$ $=16$ ). Se encontraron diferencias entre los miembros $\mathrm{E}$ y $\mathrm{O}$ afectados; los casos $\mathrm{O}$ son tres veces superiores a los $E\left(X^{2}=26.68 ; p<0.001\right)$. Si consideramos que en las enfermedades con un patrón de herencia autosómico recesivo hay un riesgo de recurrencia del $25 \%$ en la hermandad de un afectado, esto no se cumple en la genealogía de la proposita ni en esas tres genealogías, y por lo tanto no todos los casos de ACG1A se explican por este patrón de herencia. Estos datos, en su conjunto, sugieren que puede haber una mutación preferencial germinal de novo parental, en la que los padres de los pacientes son portadores asintomáticos de una mutación en estado heterocigoto, heredada por su descendencia con un patrón autosómico dominante, lo cual se ve apoyado en el presente reporte de caso, ya que en la familia hubo óbitos y algunos afectados murieron minutos después del nacimiento, como la proposita. Cuando se trata de este patrón, el riesgo de recurrencia es del 50\% o más (como se observa en la familia analizada), y este es el valor que debe usarse en el asesoramiento genético para esta patología en tales casos. El $25 \%$ es cuando se presenta en las familias con un patrón autosómico recesivo.

El mosaicismo germinal para un gen dominante podría ser otra posibilidad que explicase el patrón de la ACG1A comentada en este trabajo, ya que también ha sido reportado en la acondroplasia (ID MIM \#100800), la pseudoacondroplasia (ID MIM \#177170) y la ACG1B; displasias autosómicas dominantes en las que se han llegado a reportar casos con el mismo patrón de transmisión, como en la presente familia, en la que la recurrencia se asocia con un mosaicismo germinal ${ }^{16-20}$.

La mutación celular germinal es otra explicación, ya que la célula continúa dividiéndose en una célula somática antes de la separación en líneas celulares germinales. Considerando que en los padres de los propositi (portadores del mosaicismo) podrían observarse algunas alteraciones histopatológicas leves en el tejido conjuntivo ${ }^{18}$. Superti-Furga, et al. ${ }^{14}$ postularon que la ACG1 podría clasificarse en diferentes subgrupos. También Vargas ${ }^{21}$ reportó un caso esporádico poco común con características peculiares de ACG1A y $B$. Se ha reportado una familia en la cual los padres llevaban tres embarazos y no tenían un bebé vivo; el 
último, nacido muerto, era un feto de 24 semanas clínicamente con $\mathrm{ACG}^{2} \mathrm{~A}^{22}$. Estos antecedentes apoyan la hipótesis de que el presente caso y otros casos recurrentes de ACG1A presumiblemente corresponden a un subgrupo no descrito, debido a mosaicismo germinal o mutación preferencial germinal de novo parental.

La búsqueda de una mutación germinal o un mosaico, o una mutación preferencial germinal de novo parental en TRIP11, requiere el análisis molecular de diferentes tejidos de todos los familiares, así como el análisis de expresión génica, con biopsia e inmunohistoquímica ${ }^{14-21}$, lo cual no fue posible realizar, ya que los afectados están finados. Por cuestiones éticas, como la madre es asintomática y logró tener un hijo sano, no se aprobó la toma de biopsias, por ser además embarazos de alto riesgo. Sin embargo, puede ser parte del protocolo de diagnóstico para futuros casos de ACG1A que presenten un patrón de herencia como el que se está reportando. Los resultados de estos estudios, cuando son negativos, abren la hipótesis de otro gen causante de la ACG1A cuyas mutaciones tienen un patrón de herencia dominante, sea por una mutación de novo o germinal, e incluso el mosaico.

Tabla 2. Datos clínicos e imagenológicos comparativos en la acondrogénesis y la proposita

\begin{tabular}{|c|c|c|c|}
\hline Características clínicas & Tipo IA* & Tipo $\mathrm{IB}^{\dagger}$ & Proposita \\
\hline Polihidramnios & $18 / 22$ & $5 / 24$ & + \\
\hline \multicolumn{4}{|l|}{ Edad gestacional } \\
\hline A término & $1 / 22$ & $4 / 25$ & \\
\hline Prematurez & 20/22 & $16 / 25$ & + \\
\hline \multicolumn{4}{|l|}{ Edad al fallecimiento } \\
\hline Nacido muerto & $15 / 22$ & $11 / 25$ & \\
\hline Minutos & $11 / 22$ & $13 / 25$ & + \\
\hline Longitud al nacimiento (cm) & $22-30$ & $23.5-30$ & 23 \\
\hline Peso al nacimiento (g) & $520-1840$ & $1020-2200$ & 1600 \\
\hline Relación de sexos (masculino/femenino) & $15: 7$ & $5: 13$ & Femenino \\
\hline Consanguinidad & 3 & 2 & - \\
\hline Miembro de familia con múltiples hermanos afectados & 9 & $5^{\ddagger}$ & + \\
\hline \multicolumn{4}{|l|}{ Hallazgos radiológicos } \\
\hline Cráneo pobremente osificado & + & + & + \\
\hline Fracturas de costilla & + & - & - \\
\hline Vértebras sin osificar & + & - & + \\
\hline Pedículos posteriores osificados & - & + & - \\
\hline Ilion & Arqueado & Crenado & Arqueado \\
\hline Isquion sin osificar & - & + & - \\
\hline Fémur & $\begin{array}{l}\text { En forma de cuña, con } \\
\text { picos metafisarios }\end{array}$ & Trapezoide & $\begin{array}{l}\text { En forma de cuña, con picos } \\
\text { metafisarios }\end{array}$ \\
\hline Tibia & $\begin{array}{l}\text { Acortada, con } \\
\text { ensanchamiento } \\
\text { metafisaria }\end{array}$ & Estrellada & $\begin{array}{c}\text { Acortada, con ensanchamiento } \\
\text { metafisario }\end{array}$ \\
\hline Peroné & $\begin{array}{c}\text { Acortado, con } \\
\text { ensanchamiento } \\
\text { metafisario }\end{array}$ & No osificado & $\begin{array}{c}\text { Acortado, con ensanchamiento } \\
\text { metafisaria }\end{array}$ \\
\hline
\end{tabular}

*Veintidós casos analizados de la referencia 17.

†Veinticuatro casos reportados en la referencia 14, más un caso reportado en la referencia 15.

抽 la referencia 20 se informa de una familia consanguínea con dos fetos (después del estudio ecosonográfico, el embarazo se terminó) 
Hasta la fecha no pueden descartarse estas teorías, ya que, al igual que cuando se trata de enfermedades por genes letales, es difícil llegar a tiempo para tomar muestras de tejidos para hacer estudios citogenéticos y moleculares. Como en el presente caso, cuando se nos informó de la paciente para que la evaluáramos, ya había fallecido a los minutos, y sus hermanos ya estaban finados. Para muchas displasias esqueléticas, el diagnóstico molecular es muy útil, pero en la ACG los estándares para su solicitud no están bien establecidos, ya que los mecanismos patogénicos no están del todo demostrados ${ }^{21}$.

En población latinoamericana son escasos los estudios de ACG. Se han descrito pocos casos, entre ellos una recién nacida mexicana que llegó a las 38 semanas de gestación y falleció por bradicardia e insuficiencia respiratoria; por clínica e inmunohistopatología se estableció el diagnóstico de ACG2 (Langer-Saldino ${ }^{23}$. En población venezolana se reportó un óbito de 23 semanas, de sexo femenino, con hydrops fetalis, higroma quístico occipital, hipertelorismo, micromelia y polihidramnios, en el que por clínica, imagenología, citogenética e histopatología se concluyó ACG1B, así como un caso de un subtipo con ACG y características peculiares ${ }^{21,24}$.Con un enfoque muy similar se reportó otro caso esporádico de sexo femenino de población colombiana, gravemente afectado con ACG1A, de 23 semanas de gestación, que se interrumpió por ser un embarazo de alto riesgo ${ }^{25}$. El presente caso es el primero en población mexicana con ACG1A y el primer reporte de la literatura que presenta una ACG1A con un patrón de herencia que no es autosómico recesivo.

Desde el punto de vista histopatológico puede establecerse el diagnóstico diferencial (Tabla 3). Se ha demostrado en modelos múridos transgénicos que el fenotipo esquelético de la ACG1A es causado exclusivamente por defectos en el desarrollo del cartílago, aunque el desarrollo óseo también es anormal como consecuencia del defecto del cartílago, ya que la condrogénesis está estrechamente relacionada con la osteogénesis ${ }^{26}$. Esto explica por qué en la biopsia del fémur del caso índice con ACG1A se encontraron lagunas óseas alargadas y una zona pseudomesenquimatosa entre el cartílago y el hueso, así como cartílago extraesquelético involucrado. Estos hallazgos no han sido reportados previamente. También se ha reportado que el cartílago y el hueso son tejidos altamente secretores, con un alto tráfico de moléculas por las membranas, afectando el tráfico de un subconjunto de proteínas expresadas en los condrocitos y dando lugar a un tráfico de membrana que perjudica globalmente. Entre las moléculas que se ven afectadas están los glucosaminoglucanos sulfatados ${ }^{26}$ (que conforman parte de la matriz extracelular y la cápsula de los condrocitos). Esto explica por qué la biopsia del caso índice muestra que la cápsula de los condrocitos está más delgada (Fig. 3).

También se ha reportado en biopsias de casos de ACG1A, mediante inmunohistoquímica, que las moléculas de la matriz presentan un patrón de distribución normal, excepto la colágena tipo $X$, lo que resulta en la alteración del ensamblaje de la matriz cartilaginosa con los glucosaminoglucanos sulfatados $^{27}$. Presumiblemente, las consecuencias de este evento son los procesos de maduración y diferenciación de los condrocitos y la formación de hueso endocondral, que se ven gravemente afectados ${ }^{27}$. También se ha descrito la presencia de cuerpos de inclusión, condrocitos vacuolados hipertróficos y placas desorganizadas de condrocitos ${ }^{25-29}$; todos estos hallazgos son similares a los encontrados en la biopsia de la proposita, excepto que no se observaron cuerpos de inclusión, hallazgo que no ha sido descrito previamente. Los cuerpos de inclusión ausentes pueden explicarse por la cantidad reducida de colágena y proteoglucanos intracelularmente, lo cual es concluyente del grave trastorno de la formación endocondral. Considerando todos estos hallazgos histopatológicos, se podría proponer al gen COL10A1 (que codifica a la colágena 10) como posible gen implicado en la ACG1A con un patrón no recesivo o recurrente, como en la familia analizada del presente caso, lo cual se ve apoyado considerando que las mutaciones en COL10A1 se transmiten con un patrón dominante (herencia en la cual caben los casos de una mutación germinal o mosaico, o mutación preferencial germinal de novo parental) y producen la condrodisplasia metafisaria tipo Schimd. ${ }^{30}$ En este sentido, la ACG1A recurrente no recesiva podría ser una variante alélica del gen COL10A1, un caso muy similar con la acondroplasia (producida por mutaciones en el gen FGFR) y el enanismo tanatofórico, que es una variante alélica del mismo gen. En este trabajo no fue posible continuar con el estudio molecular TRIP11 y COL10A, ni con el análisis histopatológico, por la no biodisponibilidad de tejidos. Se realizó la tinción tricrómica de Masson para diferentes cortes del fémur del caso índice, lo que nos permitió escanear la estructura de las fibras de colágena de la matriz cartilaginosa en general, la cual no presentaba 
Cirugía y Cirujanos. 2018;86

Tabla 3. Comparación morfopatológica de los tipos de acondrogénesis

\begin{tabular}{|c|c|c|c|}
\hline Variantes de acondrogénesis & $\begin{array}{l}\text { Hallazgos histopatológicos reportados en la } \\
\text { acondrogénesis }\end{array}$ & $\begin{array}{l}\text { Hallazgos histopatológicos } \\
\text { patognomónicos }\end{array}$ & Ref. \\
\hline $\begin{array}{l}\text { ACG1A, Houston-Harris/ } \\
\text { acondroplasia rara sumamente } \\
\text { letal }\end{array}$ & $\begin{array}{l}\text { Condrocitos densamente empaquetados con } \\
\text { apariencia de ojos de toro, grandes inclusiones } \\
\text { redondas u ovales, PAS positivos, encontrando } \\
\text { dentro vacuolas adheridas a la membrana celular } \\
\text { Matriz cartilaginosa normal monocromática, } \\
\text { con moderada fibrosis perivascular y placas de } \\
\text { crecimiento desorganizadas con condrocitos } \\
\text { hipertróficos, que se extienden hacia las trabéculas } \\
\text { primaria y secundaria } \\
\text { Los condrocitos muestran signos histomórficos } \\
\text { de retención intracelular de material proteínico, } \\
\text { cantidad reducida de proteoglicanos, con } \\
\text { adelgazamiento de las fibras de colágeno } \\
\text { Falta de organización de zonas columnares de } \\
\text { condrocitos en las placas de crecimiento } \\
\text { En la microscopia electrónica se pueden apreciar } \\
\text { el retículo endoplásmico y el aparato de Golgi } \\
\text { dilatados. También se observa la expansión de las } \\
\text { cisternas del aparato de Golgi } \\
\text { Por inmunohistoquímica se ha reportado patrón } \\
\text { de distribución normal de colágenos, agrecano, } \\
\text { matrilinas, proteína oligomérica del cartílago } \\
\text { y reducción de la expresión de colágena tipo } \\
10 \text { (COL10A1) }\end{array}$ & $\begin{array}{l}\text { Metáfisis anormal } \\
\text { Condrocitos PAS positivos con } \\
\text { inclusiones citoplasmáticas } \\
\text { Los condrocitos hipertróficos semejan } \\
\text { ojos de toro }\end{array}$ & $\begin{array}{c}20-23 \\
29\end{array}$ \\
\hline
\end{tabular}

ACG1B, Fraccaro

ACG2, Langer-Saldino/ acondrogénesis letal
Desorganización de los condrocitos, los cuales muestran un incremento en el tamaño y la cantidad (hipercelularidad)

Marcada pérdida de la matriz cartilaginosa e irregularidad en la línea columnar de osificación endocondral, esclerosis mutilfocal y calcificación de la matriz

Anillos de colágena pericondrocíticos

Pérdida de la osificación significativa de la osificación e irregular en los diferentes huesos El tejido condro/óseo muestra una densidad celular alta y una matriz extracelular reducida Las metáfisis muestran osificación endocondral desorganizada con condrocitos en forma de globo; periostio con avanzada osificación, matriz condroide y lagunas de los condrocitos alargadas Los condrocitos están aumentados en número y tamaño, las columnas en la zona crecimiento están dispuestas en forma irregular

Depósito de osteoide sin calcificación, la matriz condroide presenta canales vasculares e hipervascularidad del cartílago Las trabéculas primarias son delgadas e irregulares, y fibrosis perivascular Sin inclusiones en los condrocitos Penetración vascular irregular en la línea de osificación Inmunohistoquímica de apoptosis: BAK 80\%, BAX $80 \%$ y Bcl-X $10 \%$ Inmunohistoquímica de proliferación celular: PCNA (antígeno de proliferación celular) focalmente positivo
Metáfisis anormal

Anillos de colágena pericondrocíticos

Metáfisis anormal

Los condrocitos tienen forma de globo Lagunas de condrocitos marcadamente agrandadas 
alteraciones, pero mediante esta tinción no puede explorarse particularmente la colágena tipo 10 , lo cual es una limitante de este trabajo.

\section{Conclusiones}

Mediante estudio clínico, imagenológico y anatomopatológico se presenta el primer reporte de un nuevo subgrupo de ACG1 que corresponde a la ACG1A, pero con un patrón de herencia alternativo. Ciertamente, la herencia autosómica recesiva ha sido reportada en la ACG1A, pero se descartó en el presente reporte. En futuros casos de ACG1A con este patrón alternativo de herencia, o en casos recurrentes, es conveniente hacer la búsqueda de mutaciones en el gen COL10A1 mediante secuenciación exómica. De esta manera, este es el primer trabajo en población mexicana con ACG1A y el primero de la literatura que presenta una ACG1A con un patrón de herencia que no es el autosómico recesivo. No fue posible concluir si corresponde a un defecto exclusivamente germinal o bien a un mosaicismo somático/germinal en TRIP11 y COL10A, o en algún otro gen, porque se requiere la disponibilidad de varios tejidos de la familia (de los afectados, todos finados, y de los sanos asintomáticos) para hacer la detección de mutaciones, así como realizar el análisis de la expresión génica, lo cual estuvo limitado por cuestiones éticas hospitalarias, que están en relación con el beneficio del paciente.

\section{Agradecimientos}

A M.C. Rogelio Troyo-Sanromán, por su apoyo en el análisis estadístico. A la Dra. Lina S. Correa-Cerro por la gentileza que tuvo de leer el manuscrito y expresar algunas opiniones.

\section{Responsabilidades éticas}

Protección de personas. Los autores declaran que los procedimientos seguidos se conformaron a las normas éticas del comité de experimentación humana responsable y de acuerdo con la Asociación Médica Mundial y la Declaración de Helsinki.

Confidencialidad de los datos. Los autores declaran que han seguido los protocolos de su centro de trabajo sobre la publicación de datos de pacientes.

Derecho a la privacidad y consentimiento informado. Los autores han obtenido el consentimiento informado de los pacientes y/o sujetos referidos en el artículo. Este documento obra en poder del autor de correspondencia.

\section{Financiamiento}

No existió financiación por parte de ninguna institución. Los padres de la paciente y el grupo de trabajo financiaron los estudios realizados.

\section{Conflicto de intereses}

Ninguno de los autores tiene conflictos de intereses.

\section{Bibliografía}

1. Unger S, Lachman RS, Rimoin DI. Chondrodysplasias. En: Rimoin D, Pyeritz R, Korf B, editores. Emery and Rimoin's Principles and practice of medical genetics. $5^{\text {th }}$ ed. Edinburgh: Churchill Livingstone Elsevier; 2006. p. 3709.

2. Horton WA. Abnomalities of bone estructure. En: Rimoin D, Pyeritz R, Korf B, editores. Emery and Rimoin's Principles and practice of medical genetics. $5^{\text {th }}$ ed. Edinburgh: Churchill Livingstone Elsevier; 2006. p. 3754

3. Batge B, Nerlich A, Brenner R, Yang C, Müller PK. Collagen type II in Langer Saldino achondrogenesis: absence of major abnormalities in a less severe case. Acta Paediatr. 1992;81:158-62.

4. Grigelioniene G, Geiberger S, Papadogiannakis N, Makitie O, Nishimura G, Nordgren A, et al. The phenotype range of achondrogenesis $1 \mathrm{~A}$. Am J Med Genet Part A. 2013;161A:2554-8.

5. Kapur RP. Achondrogenesis. Pediatr Dev Pathol. 2007;10:253-5.

6. OMIM. Online Mendelian Inheritance in Man, OMIM (TM). McKusick-Nathans Institute for Genetic Medicine, Johns Hopkins University (Baltimore, MD) and National Center for Biochnology Information, National Library of Medicine (Bethesda, MD). 2016. (Consultado el 10 de noviembre de 2017.) Disponible en: https://www.omim.org

7. Chen CP, Liu FF, Jan SW, Lin YN, Lan CC. A case of achondrogenesis type 1A with an occipital encephalocele. Genet Couns. 1996;7:193-9.

8. HastbackaJ, de la Chapelle A, Mahtani MM, Clines G, Reeve-Daly MP, Daly M, et al. The diastrophic dysplasia gene encodes a novel sulfate transporter: positional cloning by fine-structure linkage disequilibrium mapping. Cell. 1994;78:1073-87.

9. Ornoy A, Sekeles E, Smith P, Simkin A, Kohn G. Achondrogenesis type I in three sibling fetuses. Scanning and transmission electron microscopic studies. Am J Pathol. 1976;82:71-84.

10. Korkko J, Cohn DH, Ala-Kokko L, Krakow D, Prockop DJ. Widely distributed mutations in the COL2A1 gene produce achondrogenesis type II/ hypochondrogenesis. Am J Med Genet. 2000;92:95-100.

11. Cormier-Daire V, Savarirayan R, Lachman RS, Neidich JA, Grace K, Rimoin DL, et al. "Baby rattle" pelvis dysplasia. Am J Med Genet. 2001; 100:37-42.

12. Rossi A, Superti-Furga A. Mutations in the diastrophic dysplasia sulfate transporter (DTDST) gene (SLC26A2): 22 novel mutations, mutation review, associated skeletal phenotypes, and diagnostic relevance. Hum Mutant. 2001;17:159-71.

13. Smits $P$, Bolton AD, Funari V, Hong M, Boyden ED, Lu L, et al. Lethal skeletal dysplasia in mice and humans lacking the golgin GMAP-210. N Engl J Med. 2010;362:206-16.

14. Superti-Furga A, Hastbacka J, Rossi A, van der Harten JJ, Wilcox WR, Cohn $\mathrm{DH}$, et al. A family of chondrodysplasias caused by mutations in the diastrophic dysplasia sulfate transporter gene and associated with impaired sulfation of proteoglycans. Ann N Y Acad Sci. 1996;785:195-201.

15. Karniski LP. Functional expression and cellular distribution of diastrophic dysplasia sulfate transporter (DTDST) gene mutations in HEK cells. Hum Mol Genet. 2004;13:2165-71.

16. Feshchenko SP, Krasnopol'skaia KD, Rebrin IA, Rudakov SS. Molecular heterogeneity of proteoglycan aggregates of human hyalin cartilage in normal conditions and in systemic bone dysplasia. Vopr Med Khim. 1989;35:24-33.

17. Borochowitz Z, Lachman R, Adomian G, Spear G, Jones K, Rimoin DL. Achondrogenesis type I: delineation of further heterogeneity and identification of two distinct subgroups. J Pediatr. 1988;112:23-31.

18. Zlotogora J. Germ line mosaicism. Hum Genet. 1998;102:381-6. 
19. Comstock JM, Putnam AR, Sangle N, Lowichik A, Rose NC, Opitz JM Recurrent of achondrogenesis type 2 in sibs: additional evidence for germinale mosaicism. Am J Med Genet Part A. 2010;152A:1822-4

20. Freisinger $P$, Stanescu J, Jacob B, Cohen-Solal L, Maroteaux $P$, Bonaventure $\mathrm{J}$. Achondrogenesis type IB (Fraccaro): study of collagen in the tissue and in chondrocytes cultured in agarose. Am J Med Genet. 1994;49:439-46.

21. Vargas AJ. Valoración genética de las displasias esqueléticas. Valencia, Venezuela: Universidad de Carabobo; 1994. p. 433.

22. Oztas S, Dogan H, Kadanli S, Tatar AG. Achondrogenesis type $1 \mathrm{~A}$ (Houston-Harris). A case diagnosed at prenatal period. Gazi Medical J. 2002;13:149-51.

23. De Anda PA, Durán-Padilla MA. Acondrogénesis tipo II (Langer-Saldino). Estudio morfológico e inmunohistoquímico. Rev Med Hosp Gin Mex 2005;68:86-9

24. León de Pérez M, Velazco J, Estrada-Corona P, Puerta-Rodríguez H, Pérez-León J, Pérez- León, et al. Hallazgos ecosonográficos, citogenéticos y anatomopatológicos en feto con acondrogénesis tipo 1B. Boletín Médico de Postgrado. 2005;21(2).
25. Acosta-Guio JC, Olaya M, Zarante MI. Acondrogénesis tipo IA: reporte de caso. Rev Colom Radiol. 2011;22:3189-92.

26. Bird IM, Kim SH, Schweppe DK, Caetano-Lopes J, Robling AG, Charles JF, et al. The skeletal phenotype of achondrogenesis type $1 \mathrm{~A}$ is caused exclusively by cartilage defects. Development. 2018 Jan 8;145(1). pii: dev156588.

27. Aiger T, Rac T, Niederhagen M, Zaucke F, Schmitz M, Pöhls U, et al. Achondrogenesis type 1A (Houston-Harris): a still-unresolved molecular phenotype. Pediatr Dev Pathol. 2007;10:328-34.

28. Roseberg AE, Krishnastty NG. Skeletal system. En: Gilbert-Barnes E, editor. Potters's Pathology of the fetus, infant and child. $2^{\text {nd }}$ ed. Maryland: Mosby/Elsevier; 2007. p. 1797-897.

29. Parwanto MLE. The genetic aspect and morphological appearance of achondrogenesis. Int J Reprod Contracept Obstet Gynecol. 2017;6: 3203-12.

30. Warman ML, Abbott M, Apte S, Hefferon T, Mclntosh I, Cohn D, et al. A type $\mathrm{X}$ collagen mutation causes Schmid metaphyseal chondrodysplasia. Nature Genet. 1993;5:9-82 